\title{
Coinciding Decreases in Discharge Rate Suggest That Spontaneous Pauses in Firing of External Pallidum Neurons Are Network Driven
}

\author{
Eitan Schechtman, ${ }^{1}$ Avital Adler, ${ }^{1}$ Marc Deffains, ${ }^{1,2}$ Hila Gabbay, ${ }^{2}$ Shiran Katabi, ${ }^{2}$ Aviv Mizrahi, ${ }^{2}$ \\ and ${ }^{\circ H a g a i}$ Bergman ${ }^{1,2}$ \\ ${ }^{1}$ Edmond and Lily Safra Center for Brain Sciences, The Hebrew University, Jerusalem 91904, Israel, and ${ }^{2}$ Department of Medical Neurobiology, Institute for \\ Medical Research Israel-Canada, Hebrew University-Hadassah School of Medicine, Jerusalem 91120, Israel
}

\begin{abstract}
The external segment of the globus pallidus (GPe) is one of the core nuclei of the basal ganglia, playing a major role in normal control of behavior and in the pathophysiology of basal ganglia-related disorders such as Parkinson's disease. In vivo, most neurons in the GPe are characterized by high firing rates ( $50-100$ spikes/s), interspersed with long periods $(\sim 0.6 \mathrm{~s})$ of complete silence, which are termed GPe pauses. Previous physiological studies of single and pairs of GPe neurons have failed to fully disclose the physiological process by which these pauses originate. We examined 1001 simultaneously recorded pairs of high-frequency discharge GPe cells recorded from four monkeys during task-irrelevant periods, considering the activity in one cell while the other is pausing. We found that pauses $(n=137,278$ pauses $)$ coincide with a small yet significant reduction in firing rate $(0.78 \pm 0.136$ spikes $/ \mathrm{s})$ in other GPe cells. Additionally, we found an increase in the probability of the simultaneously recorded cell to pause during the pause period of the "trigger" cell. Importantly, this increase in the probability to pause at the same time does not account for the reduction in firing rate by itself. Modeling of GPe cells as class 2 excitability neurons (Hodgkin, 1948) with common external inputs can explain our results. We suggest that common inputs decrease the GPe discharge rate and lead to a bifurcation phenomenon (pause) in some of the GPe neurons.
\end{abstract}

Key words: basal ganglia; globus pallidus; network; pause

\section{Introduction}

The external segment of the globus pallidus (GPe) is one of the core nuclei of the basal ganglia. Receiving input mainly from the striatum and the subthalamic nucleus (STN), and projecting to the output nuclei of the basal ganglia, it is hypothesized as being the main processing site of this complex neural structure (Goldberg and Bergman, 2011; Jaeger and Kita, 2011; Gittis et al., 2014). Most GPe neurons fire at a rate of 50-100 spikes/s, although some fire at a significantly lower pace $(<20 \mathrm{~Hz})$. The high-frequency discharge (HFD) neurons of the GPe are characterized by a unique feature:

Received Dec. 24, 2014; revised Feb. 25, 2015; accepted March 17, 2015

Author contributions: E.S. and H.B. designed research; E.S., A.A., M.D., H.G., S.K., and A.M. performed research; E.S. analyzed data; E.S. and H.B. wrote the paper.

This work was supported by research grants from the European Research Council, the Israel Science Foundation, the United States-Israel Binational Science Foundation, the German-Israeli Foundation for Scientific Research and Development, the Adelis Foundation, the Rosetrees Trust, the Vorst Foundation, and the Simone and Bernard Guttman Chair in Brain Research at The Hebrew University, Jerusalem, Israel. E.S. is a recipient of the Harry and Silvia Hoffman leadership program fellowship and of the Adams Fellowship Program of the Israel Academy of Sciences.

The authors declare no competing financial interests.

This article is freely available online through the $J$ Neurosci Author Open Choice option.

Correspondence should be addressed to Eitan Schechtman, Edmond and Lily Safra Center for Brain Sciences, The Hebrew University, Jerusalem 91904, Israel. E-mail: eitan.schechtman@mail.huji.ac.il.

A. Adler's present address: Skirball Institute, Molecular Neurobiology Program and Department of Physiology and Neuroscience, NYU School of Medicine and Center for Neural Science, New York University, New York, NY 10016. DOI:10.1523/JNEUROSCI.5232-14.2015

Copyright $\odot 2015$ Schechtman et al.

This is an Open Access article distributed under the terms of the Creative Commons Attribution License Creative Commons Attribution 4.0 International, which permits unrestricted use, distribution and reproduction in any medium provided that the original work is properly attributed. they often pause their firing completely for periods of several hundreds of milliseconds (DeLong, 1971; Elias et al., 2007).

GPe pauses have intrigued basal ganglia physiologists for decades due to their prominence $(>56 \%$ of HFD GPe neurons exhibit pausing behavior; Elias et al., 2007) and distinct dichotomic nature. However, questions regarding the origin of pauses and their functional importance have seldom been addressed. In a series of studies, Kita et al. (2004, 2005, 2006), Kaneda and Kita (2005), and Hashimoto and Kita (2006) have shown that pause generation depends on complex patterns of interaction between GABAergic and glutamatergic inputs. Together with the anatomy and synaptic interactions of GPe cells (Difiglia et al., 1982; Yelnik et al., 1984; Shink and Smith, 1995; Nambu et al., 2000; Sato et al., 2000; Mallet et al., 2012), these findings suggest that GPe pauses depend on the interaction of GABAergic inputs from the striatum or other GPe cells, and the glutamatergic inputs from the subthalamic nucleus.

If, indeed, pauses were a network phenomenon, depending mainly on input from other basal ganglia structures, there should be pairwise correlations between sets of GPe neurons that share common inputs. However, this does not seem to be the case. Elias et al. (2007) investigated the hypothesis that pauses are correlated in some of their aspects. They found no indication for pairwise correlations between pause times among simultaneously recorded neurons (including neurons recorded from the same electrode), suggesting that neurons generate pauses in an independent manner. Unlike the previously mentioned in vitro studies, which found that 


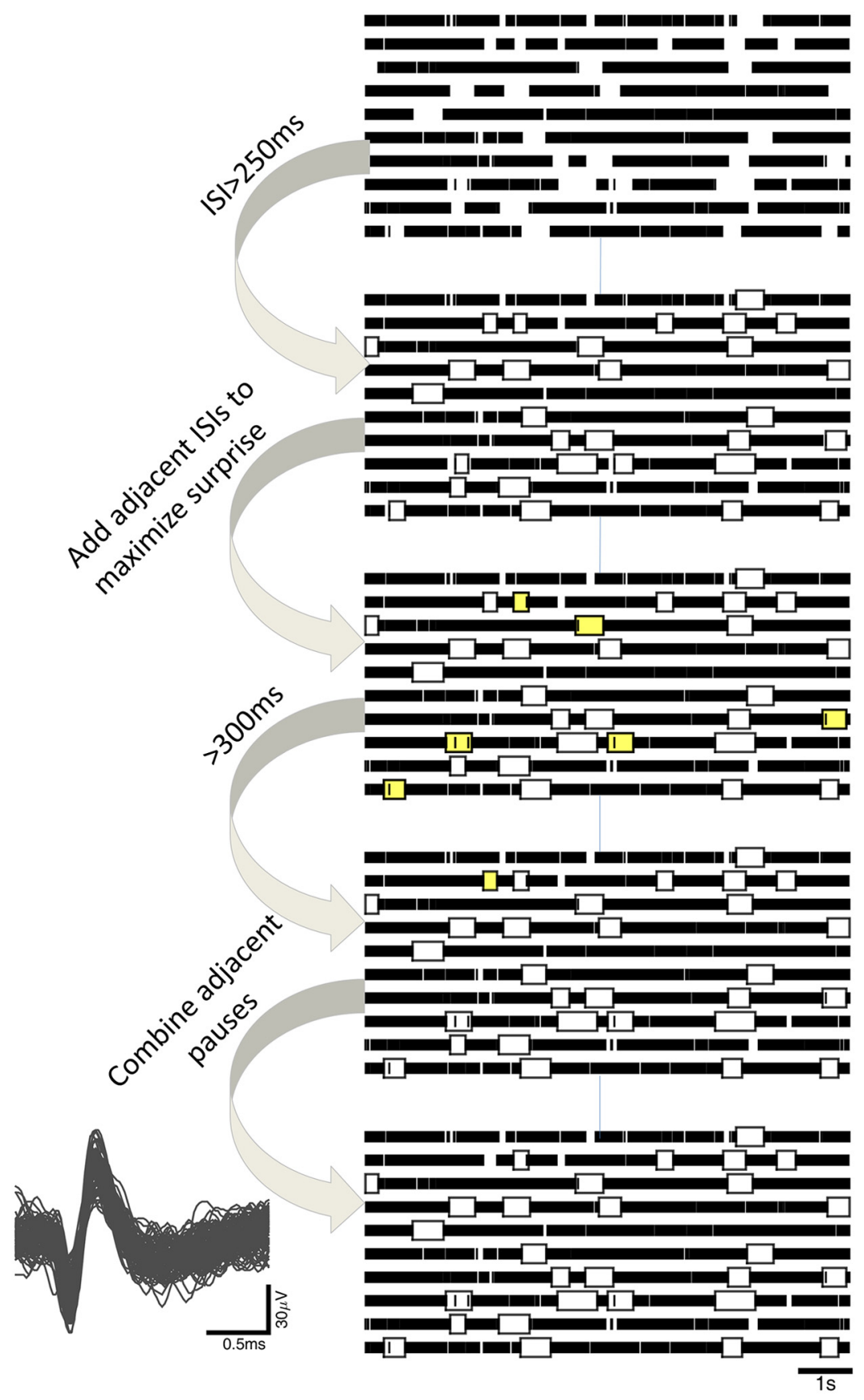

Figure 1. Pause detection method. All raster plots show the firing activity of a single GPe neuron over 10 consecutive $10 \mathrm{~s} \mathrm{time}$ windows (Monkey C, recorded November 28, 2013; isolation score $=0.88$; bottom-left inset shows 100 superimposed spikes). The different panels, from top to bottom, show the sequential steps of the pause detection algorithm and all segments identified as pauses at each step. Changes from the previous step are indicated in yellow. The bottom panel shows the final result of the pause detection algorithm for this neuron during these time segments.

pauses rely on external input to the GPe, this finding was revealed in a spontaneously untampered system in vivo, providing a more naturalistic outlook.

In this study, we aimed to re-examine the issue of intrinsic versus network-driven pauses. We established a genuine approach for the analysis of the network activity patterns giving rise to pauses. Using data from well isolated extracellularly recorded single units from four different monkeys, we examined the simultaneous population activity of pairs of GPe HFD neurons during time intervals in which at least one of the pair was pausing. This method is sensitive to very small effects, which might have been dismissed using other approaches oriented to pairwise behavior. Our findings shed new light on the origin of pauses and on the functional connectivity of the basal ganglia circuitry.

\section{Materials and Methods}

Animals and procedures. Four monkeys [1 male; 2 Macaque fascicularis and 2 Chlorocebus aethiops (African green monkeys); weight range, $3-4.6 \mathrm{~kg}$ ] were used in this study. Experimental protocols were conducted in accordance with the National Research Council Guide for the Care and Use of Laboratory Animals and the Hebrew University guidelines for the use and care of laboratory animals in research. The experimental protocols were approved and supervised by the Institutional Animal Care and Use Committee of the Hebrew University and Hadassah Medical Center. The Hebrew University is an Association for Assessment and Accreditation of Laboratory Animal Care internationally accredited institute. The surgery procedures and datarecording methods were described in previous articles (Adler et al., 2012, 2013a,b). Extracellular recording of spiking activity was performed using up to eight glass-coated tungsten electrodes in a single session. Neurons simultaneously recorded in the GPe were no farther apart than $1.5 \mathrm{~mm}$ on the horizontal axis of the electrodes, and $1 \mathrm{~mm}$ on the vertical axis.

Pause detection. Pauses were identified using the method described in the study by Elias et al. (2007; Fig. 1). This method uses a deterministic algorithm for pause detection rather than a probabilistic one. Briefly, the procedure is based on an evaluation of how improbable (or "surprising") it is for a certain number of spikes (or fewer) to appear in a time segment, given the average firing rate (FR; Legéndy and Salcman, 1985) of the unit. Using this modified surprise method, we first identified spike-free segments for each unit. These interspikes intervals (ISIs) were at least $250 \mathrm{~ms}$ long. However, because sometimes pauses are interspersed with single spikes, the method allows for the enhancement of periods defined as pauses using the following equation:

$$
P(n)=\mathrm{e}^{-r T} \sum_{i=0}^{n}(r T)^{i} / i !
$$

where $r$ is the mean FR (in spikes per millisecond), and $P(n)$ is the probability to find $n$ spikes or fewer in an interval of $T$ milliseconds. We defined the surprise function as $S=$

$-\log (P(n))$. For each identified segment, we checked whether adding another neighboring ISI increases the surprise. If so, the pause was redefined to include this interval. A maximum of five ISIs was added for each side in this fashion. Following this procedure, pauses that did not exceed a duration of $300 \mathrm{~ms}$ were omitted. Additionally, pauses that were adjacent one to the other (i.e., divided by a single spike) were combined. The pauses detected using these criteria were shown to match those identified by human experts (Elias et al., 2007).

Inclusion criteria. Only stably held, well isolated units (isolation score $>0.8$; Joshua et al., 2007) that were unquestionably located in the GPe and fired $>20$ spikes/s on average were used. Our analysis was limited solely to units that were simultaneously recorded with other stable and 
well isolated GPe units for a minimum of $50 \mathrm{~s}$. The average simultaneous recording period for pairs of units was $1699 \mathrm{~s}$ ( $n=1001$ pairs), and repeating the analysis for pairs that were simultaneously recorded for at least $600 \mathrm{~s}$ ( $n=453$ pairs) revealed similar results.

The four monkeys participated in different tasks during the recording period (Adler et al., 2012, 2013a,b). However, because we were interested solely in spontaneous pausing, we omitted all time periods that were followed or preceded by any behavioral event in a $0.5 \mathrm{~s}$ time range. The analysis was also run with omissions of time periods in 1 and $2 \mathrm{~s}$ time ranges around behavioral events with qualitatively similar results (data not shown).

Firing rates and pause probabilities. For each identified unit, we considered all pause segments. The start points of these segments, abbreviated as trigger pauses (TPs), were used as a reference point for all other units that were simultaneously recorded during this time. Although each pair of units was considered twice (pauses in unit A acted as a TP to measure the activity of unit $B$, and vice versa), we made sure that all considered segments were independent. This was accomplished by taking into account only one segment of each pair of segments that partially overlap in time, which was chosen randomly.

The FR and pause probability [PP (defined as the probability for each time point to be included within a time segment marked as a pause)] of all these simultaneously recorded units were averaged and smoothed (50-ms-wide Gaussian window; $\mathrm{SD}=10 \mathrm{~ms})$. The period starting $2 \mathrm{~s}$ before the TP initiation and ending $1 \mathrm{~s}$ before it was defined as the baseline, and its mean was subtracted from the signal for both FR and PP.

Clearly, pauses involve a decrease in discharge rate, which influences the mean FR values. To disentangle FR decreases that resulted from pausing behavior and those that did not, we analyzed the FR dynamics, excluding all segments in which a unit was pausing. To do so, we repeated the previously mentioned analysis, omitting each time point in which a pause was detected. We defined this measure, which was also smoothed using a 50-ms-wide Gaussian window, as the "pause-omitted firing rate."

To verify that undetected pauses did not still contaminate the pauseomitted firing rate measure, we first defined the percentage of change in FR relative to baseline per pause segment as follows:

$$
C_{n}=100 * \frac{\int_{-250 m s}^{250 m s} r_{n}(t) d t / 500-\int_{-2000 m s}^{-1000 m s} r_{n}(t) d t / 1000}{\int_{-2000 m s}^{-1000 m s} r_{n}(t) d t / 1000},
$$

where $n$ is the segment number, $r_{n}(t)$ is the FR in that segment as a function of time, and $t=0$ marks the TP. The subgroup of segments that contain pauses synced with the TP (or slightly preceding or exceeding it) mostly produce values around -100 . This index was calculated, per segment, for the unaltered data and for the pause-omitted firing rate. The results for all segments were used to create two histograms, one for each aforementioned dataset. If, indeed, pauses were successfully removed in the pause-omitted data, we expected to see a difference in the lower part of the histogram, and most notably in the values around -100 (i.e., a larger probability around this value for the unaltered data relative to the pause-omitted data; see Fig. 3e).

To verify that our results are not affected by hidden biases of the analysis methods, we reconducted the analysis using data shifted in small intervals. For each unit pair considered, we shifted the TP time point back $5 \mathrm{~s}$. This relatively short time lag was used to conserve latent nonstationarities in the data. We used $t$ tests to compare the results obtained for the shifted dataset and the real results. We also conducted comparisons to data shifted using a random time lag and got similar results (data not shown).

The results were averaged across segments, regardless of units. We also conducted analyses over all units and all pairs for which there were data recorded simultaneously with at least $20 \mathrm{TPs}$, and the results were qualitatively similar (data not shown). Additionally, we conducted the same analy- sis considering the pause end (and not the pause start) as a trigger. The results obtained resembled a mirror image of those presented in this article.

Pause coincidence measures. To estimate whether the difference in the level of pause coincidence (PC) between two units was more than a chance occurrence, we first defined a PC index as the number of pauses two units initiate no more than $250 \mathrm{~ms}$ from one another, divided by the minimal number of pauses in any of the two units during their simultaneously recorded period. For example, if one unit had 20 pauses during the simultaneous recording period and the other had 30, a total of 15 pauses starting within $250 \mathrm{~ms}$ from one another will result in a PC index of $15 / 20=0.75$. The PC index is defined per unit pair. If one or more of the units had $<20$ pauses during the simultaneous recording period, their index was not calculated.

Despite the correction for pause count, the PC index still depends on the frequency of pauses (e.g., frequently pausing units will produce more random coincidences). Therefore, we conducted shifts in the data, recalculated the PC index after each shift, and compared the results. To preserve nonstationarities in the data, we shifted the data in small time bins $(0.5-15 \mathrm{~s}$ in each direction in $0.5 \mathrm{~s}$ intervals, for a total of $60 \mathrm{shifts})$ and calculated the PC index for each of these shifts (see an example in Fig. $4 b$ ). Finally, we defined a measure of significance for each pair by measuring the probability of a shift to yield a PC index that is more extreme than the results obtained for the unshifted data, as follows:

$$
\begin{array}{r}
p_{m}=\frac{\sum_{i=1}^{60} N\left(\operatorname{abs}\left(\overline{C I_{m}}-C I_{\text {real }, m}\right), a b s\left(\overline{C I_{m}}-C I_{i, m}\right)\right)}{60}, \\
N(x, y)=\left\{\begin{array}{cc}
1, & x>y \\
0, & \text { o.w }
\end{array}\right.
\end{array}
$$

where $m$ is the pair number, $\mathrm{CI}_{\text {real }, m}$ is the coincidence index for the unshifted data in pair $m, \mathrm{CI}_{i, m}$ is the coincidence index for shift number $i$ in pair $m$, and $\overline{C I_{m}}$ is the mean for all shifts. The result is equivalent to $p$ values attained using standard permutation tests.

The results for all pairs were compared with the results obtained by randomizing pause position. The same measures were calculated for the same pairs, except that pause times for each unit were randomly distributed across the simultaneously recorded period for the pair. This randomization was repeated 30 times for each pair, and the resulting measures were pooled.

This analysis was also conducted using different parameters (pauses $500 \mathrm{~ms}$ apart were considered coincident; significance was calculated using 30 shifts with $1 \mathrm{~s}$ intervals). This yielded a qualitatively similar result (data not shown).

Data analyses. Data analyses and visualization were performed using Matlab (MATLAB Release 2014b, MathWorks). All population values are given as the mean \pm SEM. Figures show the SEM as shaded regions.

\section{Results}

\section{Descriptive statistics for database}

A total of 448 GPe high-frequency discharge units, which constitute 623 pairs, met our inclusion criteria (73-136 units and 135184 pairs per monkey). We considered some pairs twice $(A \rightarrow B$, $\mathrm{B} \rightarrow \mathrm{A}$ ) but avoided double use of the simultaneous pauses (see Materials and Methods), raising the total number of analyzed pairs to 1001. The average stable recording length for these units was $2266 \pm 80.4 \mathrm{~s}$, and the average isolation quality was $0.934 \pm$ 0.0026 . The average simultaneous recording period for pairs of units was $1699 \pm 52.49 \mathrm{~s}$. The average FR of the unit was $76.79 \pm$ 1.29 spikes/s. Pauses were detected using a modified Poisson surprise method (Elias et al., 2007; Fig. 1). The recorded GPe units produced a total of 137,278 pauses, or $306.42 \pm 22.6$ pauses/ neuron during the recording period, with a mean frequency of $8.82 \pm 0.08 \mathrm{pauses} / \mathrm{min}$ and a mean pause duration of $0.6 \pm$ $0.05 \mathrm{~s}$ (typical data and pairwise analysis are shown in Fig. 2). Together, these data suggest that every single time point within 


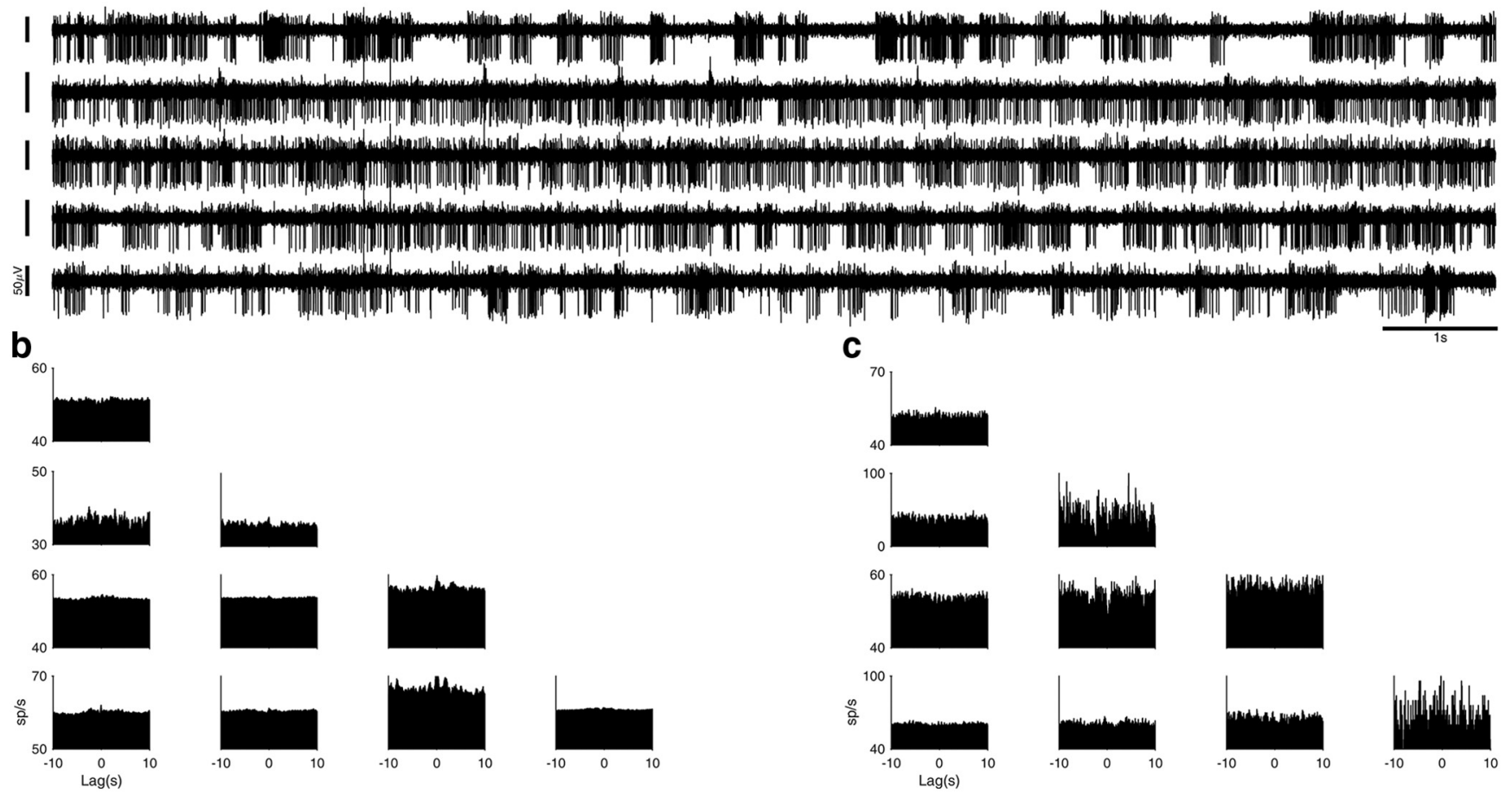

Figure 2. Examples of pausing behavior in GPe cells (Monkey C, recorded November 7, 2013). $\boldsymbol{a}$, Analog signal of five electrodes showing the spiking activity of five simultaneously recorded GPe neurons, each displaying pausing activity at different rates and lengths. The signal was digitally filtered using an eight-pole Butterworth bandpass filter between $300 \mathrm{~Hz}$ and $5 \mathrm{KHz}$. $\boldsymbol{b}$, Spike-to-spike cross-correlation diagrams for each of the 10 pairs of units shown in $\boldsymbol{a}$. Bin sizes were $50 \mathrm{~ms}$. $\boldsymbol{c}$, Spike-to-pause cross-correlation diagrams for each of the 10 pairs of units shown in $\boldsymbol{a}$. This was done by averaging the probability of spike discharge in another GPe neuron (recorded by another electrode) around the start of a pause. Bin sizes were $50 \mathrm{~ms}$.

the recording time of a unit had an $8.1 \%$ chance to fall within a pause segment.

Population firing and pausing activity during pause segments Considering the time frame of each pause, we examined the simultaneous activity of all other units. We found a small $(0.78 \pm$ 0.136 spikes/s), but statistically significant, decrease in the average FR $(p<0.001$; Fig. $3 a, b)$. This FR decrease initiated shortly before the TP starting time point (Fig. $3 a$ ) and lasted longer than the pause duration, but returned to baseline within $1 \mathrm{~s}$ after the end of the pause (Fig. $3 b$ ).

Conducting a similar analysis, we found a slight $(0.2 \%)$ increase in the PP around the TP $(p=0.027$; Fig. $3 c)$, indicating that units tended to pause together. This increase was smeared in time, indicating that the simultaneously occurring pauses were not well locked temporally. The temporal profile of the probability of pause occurrence was similar to that of the discharge rate around the TP (Fig. 3a,c).

Is the change in pause probability the sole contributor to the fall in FR? To check this, we re-examined the firing activity, this time omitting from the analysis each time point in which the unit was identified as pausing (i.e., the pause-omitted firing rate). We found that only part of the reduction in the firing rate was due to the change in pause probability, and a decrease of discharge rate is evident even after the omission of pause-contaminated segments $(p<0.001$; Fig. $3 d)$. To verify that this is not simply due to undetected pauses, we drew a histogram of the change in FR around the TP for the original data and for the pause-omitted firing rate, and observed that this procedure mostly eliminated pauses (Fig. 3e).
Probability of coinciding pauses for different unit pairs Following our observation that pauses sometimes occur together, we sought to examine whether some neuron pairs' pauses tended to coincide more than those of others. We checked all pairs of units that each had at least 20 pauses during their simultaneously recorded period ( $n=373$ pairs) and calculated the following two measures: a coincidence index, ranging from 0 to 1 , which indicates the probability of pauses of a pair of neurons to coincide (Fig. $4 a$, left); and a significance measure, which compares the index to indices achieved through data shifts (Fig. $4 a$, right). We found that, compared with shifted data, there were far more instances of high indices, meaning that the number of pairs with strong pause coincidence indices is higher than is to be expected by chance. Figure $4 b$ depicts an example of the calculation of the coincidence index and the significance for two pairs of units, one with and one without a significant $(p<0.05)$ index value. Figure $4 c$ shows the mean change in FR relative to the trigger pause for the following two subgroups of pairs: pairs that had indices that were comparable to those achieved by data shifts; and pairs with indices that were significantly higher than chance $(n=42$ and 40 , respectively). The dynamic changes in pause probability for these two groups also had greatly differing profiles (data not shown).

\section{Discussion}

Averaging the simultaneous firing activity of GPe neurons over all pauses, we observed a slight yet significant decrease in GPe FR. This effect, which was probably too small to be identified in a pairwise cross-correlation analysis that is limited to specific neuron pairs, shows that while a single GPe neuron is pausing, others are also inhibited. The simplest explanation for this could be that other neurons also paused in coincidence, and we found that 
a

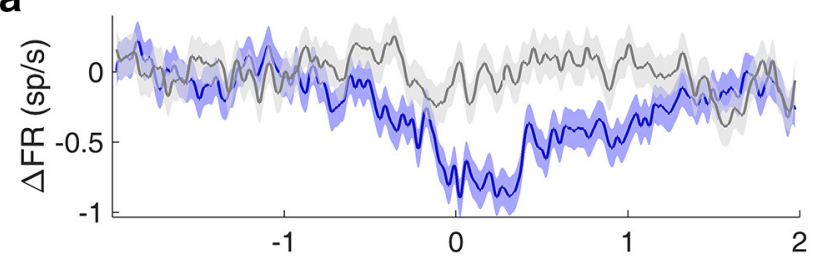

C

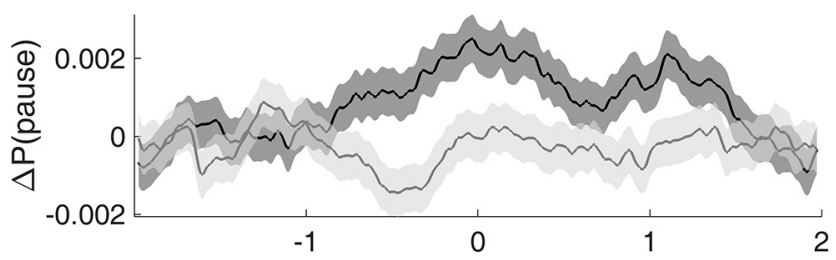

d

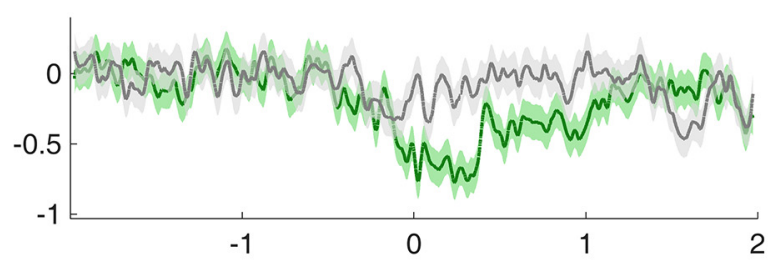

b

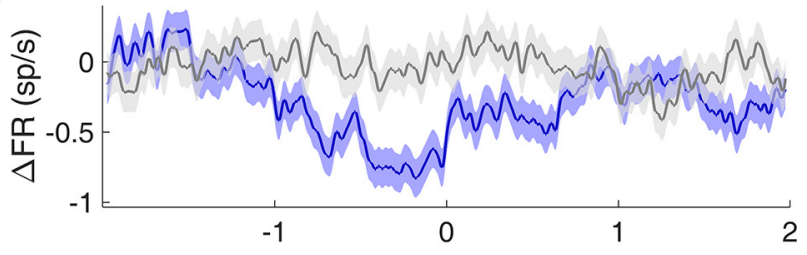

e

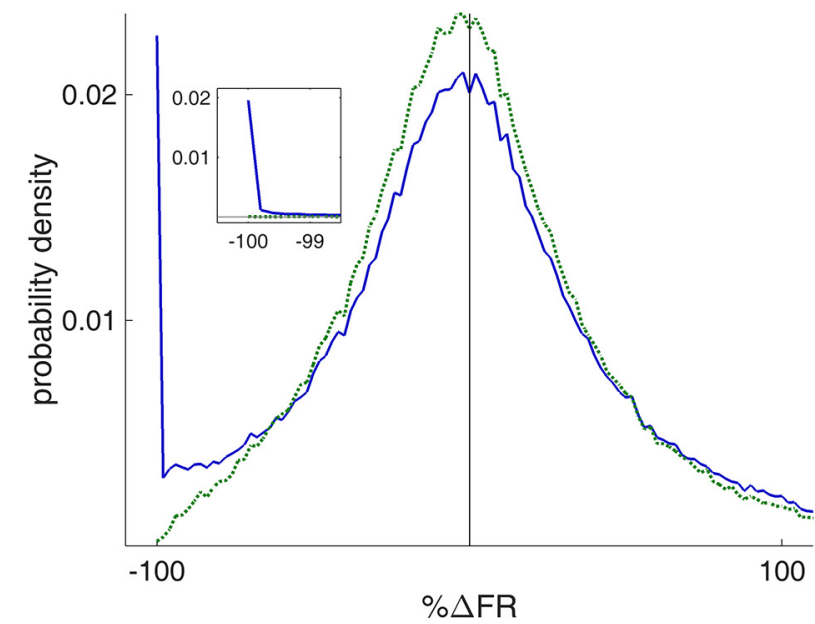

Figure 3. Population GPe firing and pausing activity triggered to a simultaneous pause. $\boldsymbol{a}$, Mean firing rate across repetitions during time segments in which other units were pausing (baseline reduced).Zero time depicts trigger pause initiation. The dark blue line signifies the real data $(n=159,906$ repetitions). The light gray line depicts the results after shifting the data. Data were smoothed using a $50 \mathrm{~ms} G$ aussian window. Surrounding shading represents the SEM. $\boldsymbol{b}$, Same as $\boldsymbol{a}$; zero time represents pause termination ( $n=159,752$ repetitions). $\boldsymbol{c}$, Change in pause probability across repetitions relative to baseline triggered by the initiation of a pause. Details follow those of $\boldsymbol{a}$. $\boldsymbol{d}$, Mean firing rate across repetitions after removal of pause-contaminated segments. Details follow those of $\boldsymbol{a}$. $\boldsymbol{e}$, Normalized histogram of changes in firing rate (per segment) in the pause-related period relative to baseline (in percentages). The blue line indicates the results of the data beforethe removal of pause-contaminated segments (i.e., the same as in $\boldsymbol{a}$ ); the green line indicates the results of the data after the removal of pause-contaminated segments (i.e., the same as in c). The inset shows a zoomed-in display of the left-most part of the plot with enhanced $x$-scale resolution.

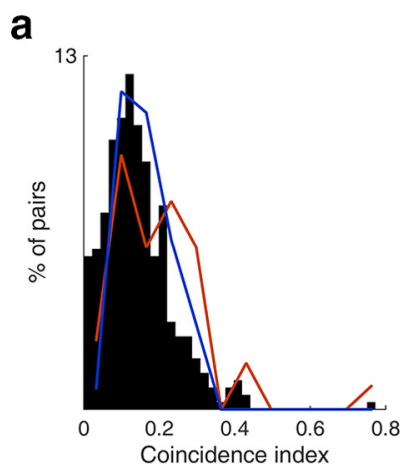

b

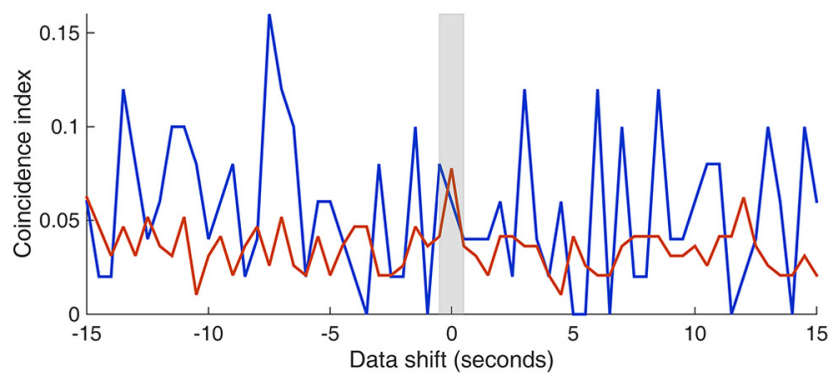

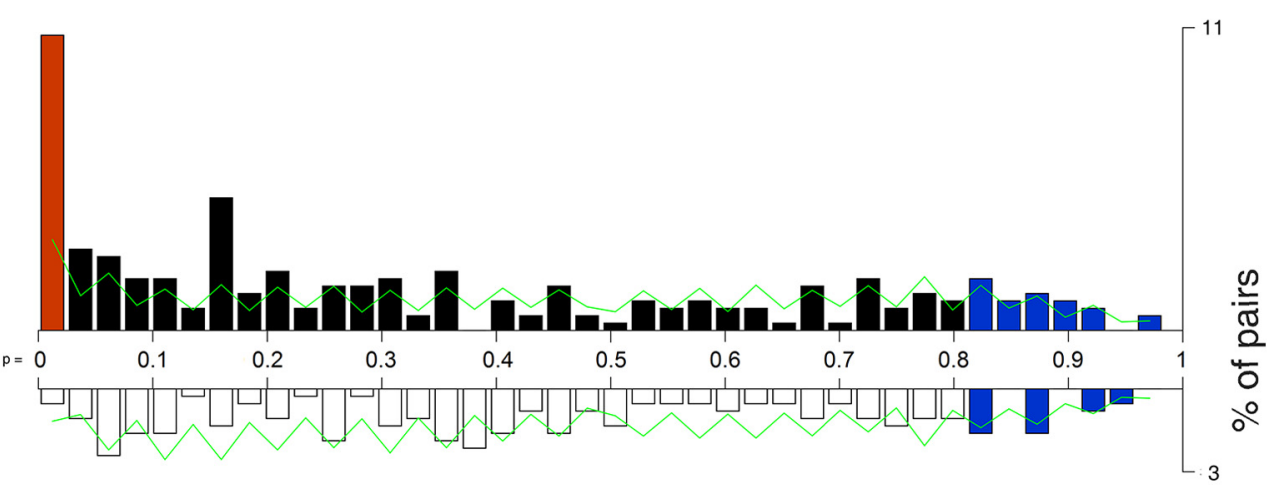

C

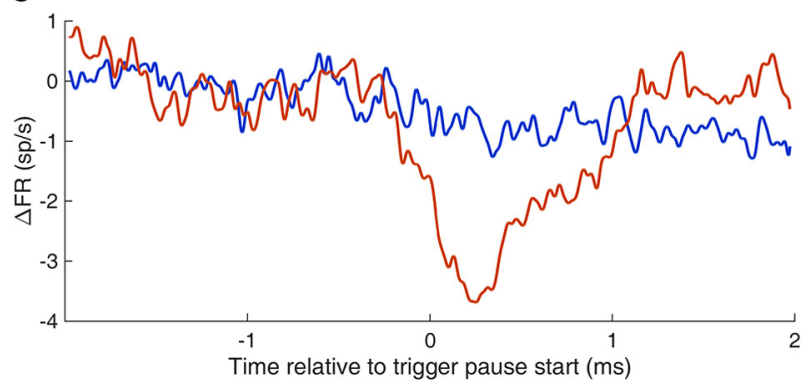

Figure 4. Measures of $\mathrm{PC}$ between pairs of neurons. $\boldsymbol{a}$, Left, Bars indicate the observed distribution of PC indices for all unit pairs. Right, Bars indicate the observed distribution of significance level ( $p$ values) for all unit pairs $(n=373)$. The $p$ values were calculated by comparing pairs' real PC index to the distribution of indices for shifted data. The black bars on top (white bars on the bottom) signify indices that were higher (lower) than the mean of this distribution, illuminating the high proportion of significant PC indices that havehigher-than-chance PC indices (red). This group is compared with a group with nonsignificant PC indices (blue) on the left and in further panels. The green line indicates the same measures for randomized data. $\boldsymbol{b}$, Example of the calculation method for two unit pairs, one taken from the blue bars and one from the red bar in $\boldsymbol{a}$. The shaded area marks the results obtained without shifting (the results for the real data). Note that the time shifts decrease the coincidence index for the red pair, but not for the blue pair. Red, coincidence index $=0.08, p=0$; blue, index $=0.06, p=0.83$. c, The mean FR relative to a trigger pause for all of the unit pairs included in the bars marked in blue and in red in $\boldsymbol{a}$. Data were smoothed using a $50 \mathrm{~ms}$ Gaussian window. As expected, the mean simultaneous activity to a trigger pause decreases for pairs with highly significant coincidence indices. 


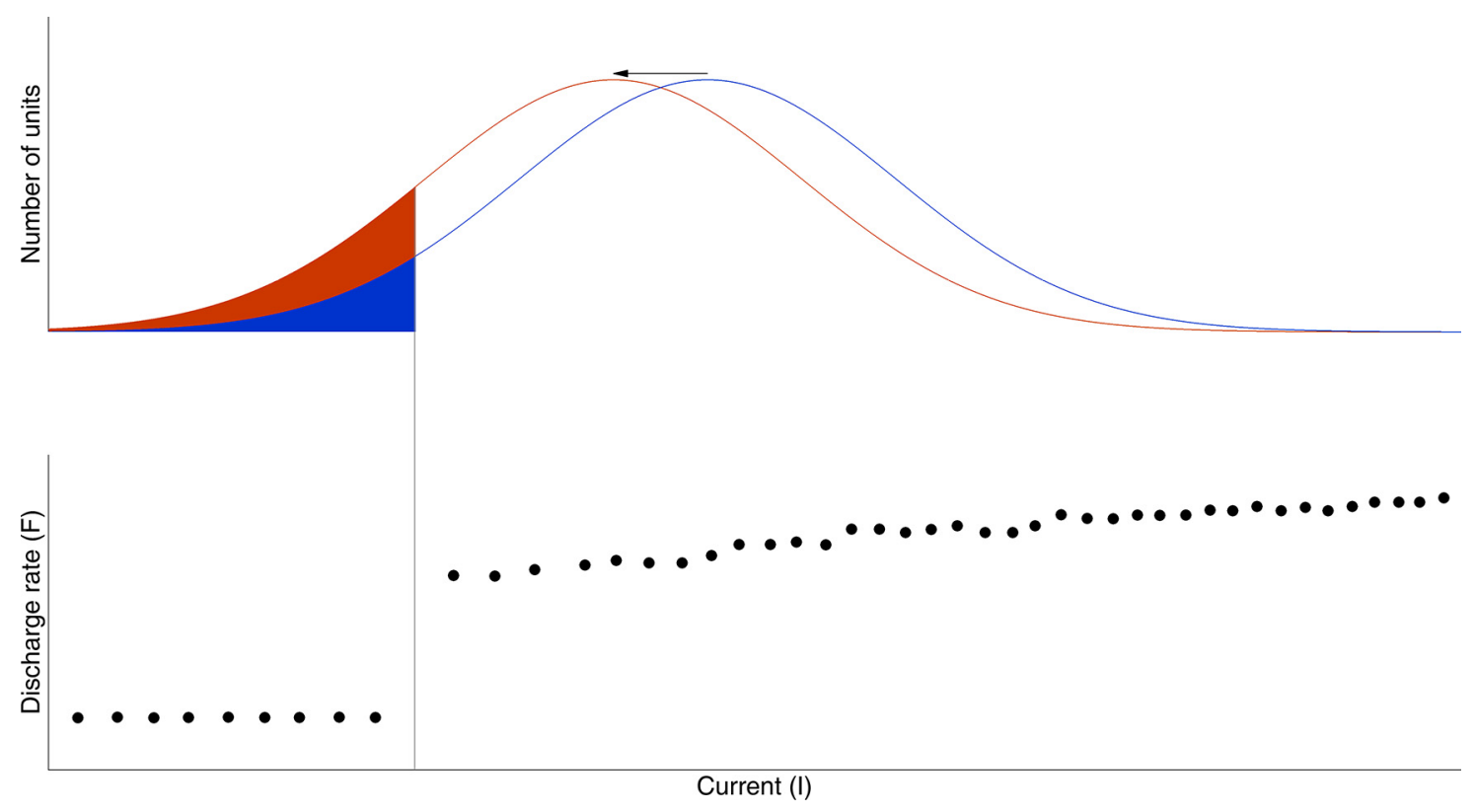

Figure 5. Modeling GPe neurons as class II excitability cells. The bottom axes show the activity of a class II excitability cell (adapted from Izhikevich, 2007). This cell does not fire until a certain threshold of inward current is reached, and then increases its firing rate in a linear fashion. The top plot shows two distributions of hypothetical GPe cells at a given time, implicating the current they are receiving. The blue line represents a hypothetical distribution, for which a certain percentage of neurons, colored in blue, are "pausing" (i.e., their predicted firing rateis 0 ). After this distribution is shifted (arrow) due to an increase in inhibitory input or a reduction in the excitatory drive, the new distribution (red line) has a lower net input current, a higher probability of cells to pause (red-colored region), and a slightly lower predicted average firing rate.

such pause coincidences do, indeed, occur. However, these coincidences did not solely account for the decrease in the GPe discharge rate, and we revealed that a decrease in the firing rate was still manifested upon omission of this pausing activity. Put together, these findings indicate that despite the diverse activity across the GPe network, there seems to be a synchronous net decrease in GPe discharge rate and rise in the general PP. The observed changes in PP and in FR do not appear to be due to the contribution of several subsets of neurons showing different activity characteristics that are synchronized with pauses. However, when considering neuron pairs, there does seem to be some nonhomogeneity. The increase in PP is not equally spread across the network-some neuron pairs tended to pause together at a higher-than-chance level (Fig. 4), while most others did not.

Our finding that pauses tend to occur together sits well with studies identifying pauses as a network phenomenon (Jaeger and Kita, 2011) and not one that is generated independently for a single neuron. The most parsimonious interpretation of our data is that there are common inputs to sets of GPe neurons, influencing their tendency to decrease their discharge rate or pause at a given time. However, it could also be that pauses are intrinsically generated within a single neuron and propagate through lateral inhibition (i.e., the "original" pause causes increases in the FR of several neurons, which in turn inhibit a second-order set of neurons, causing some of them to pause). If this was the case, we would expect the group of neurons that increase their FR to have a very low coincidence index when paired with their "pauser" cell. However, we did not see a distinct set of neurons that show lowerthan-chance probabilities to pause together (Fig. $4 a$, right, left-most white bars). The lack of such a set suggests that the lateral effects of pauses in GPe are not very strong, despite the significant intrinsic connectivity within the GPe (Sadek et al., 2007; Mallet et al., 2012).

If indeed pauses are the product of common afferent inputs, which model can best explain both the increase in simultaneous PP and the independent decrease in FR? In 1948, Hodgkin (1948) set apart the following two classes of neurons: class I excitability neurons, which increase their discharge rate linearly in response to increases in net input current; and class II excitability neurons, which act in a nonlinear fashion, abstaining from firing until a certain current threshold is crossed and then leaping to a high $(\sim 100$ spikes/s) FR, which increases linearly with current. More recently, Goldberg and Bergman (2011) suggested that the activity of GPe cells could fit the class II requirements. Other classifications of neuronal excitability have been suggested (Izhikevich, 2007), but they maintain the two dynamic schemes of class I and II excitability.

This model could explain our main findings quite elegantly (Fig. 5), assuming that at each given time the total synaptic current that each GPe neuron receives is taken from a normal distribution, the center of this distribution defines the average population current and hence the average population FR. In neurons for which the current "falls" below some threshold, a pause is observed due to the relation between net input current and FR (I-F relation) of class II excitability neurons. If the system receives widespread common inhibitory striatal input or a common reduction of the excitatory drive from the subthalamic nucleus, this distribution will shift to the left. This shift would cause an increase in pause probability (red vs blue filled areas) and a decrease in FR (evident by the FR expected for the new histogram). Therefore, this model predicts that conditions that cause decreases in FR will also increase pause probability, despite pauses being a phenomenon distinct from discharge rate reduction. This sits well with the finding that pause probability rises upon hyperpolarization of rodent GPe cells in vitro (Hashimoto and Kita, 2006), and the tendency of GPe neurons to reduce their FR and also increase their pause probability when monkeys close their eyes (Adler et al., 2010).

On top of the implications this model has for the activity of the $\mathrm{GPe}$, it might also prove useful in examining the differences between neurons of the GPe and the internal segment of the globus pallidus (GPi). Neurons of the GPi are morphologically similar to those of the GPe (François et al., 1984; Yelnik et al., 1984), and 
both receive synaptic inputs from striatal medium spiny neurons (MSNs), STN cells, and GPe cells, albeit that the contributions of these inputs are not the same in these two structures (Shink and Smith, 1995). However, neurons of the GPi commonly have higher firing rates than those of the GPe (DeLong, 1971; DeLong et al., 1985). Pauses are mostly restricted to the GPe, but are also sparsely observed in the GPi (DeLong et al., 1985; Elias et al., 2007; Chiken et al., 2008). These properties, together with our model, raise the possibility that GPi cells could be similarly modeled as class II neurons. Perhaps it is a different input current that sets these two neuron populations apart, thus explaining both the higher firing rate and the lower pause probability in GPi cells. This hypothesis suits recent data showing that the group of inhibitory MSNs targeting the GPi (which commonly have $\mathrm{D}_{1}$ but not $\mathrm{D}_{2}$ receptors; Gerfen et al., 1990) has a lower excitability level than that targeting GPe, potentially contributing to the higher firing rates of GPi neurons (Day et al., 2008; Surmeier et al., 2011).

The simultaneous increase in pause probability in the GPe is most likely due to the cluster of neuron pairs that show a higher than chance likelihood to pause together (Fig. 4). Using this cluster, we can estimate measures of connectivity in the network. Of the 373 pairs passing our inclusion criteria (see Materials and Methods), $\sim 40$ (10.7\%) showed higher than chance pause coincidence. Monkeys have $\sim 166,000$ GPe neurons (Percheron et al., 1994), which constitute $13.8 \times 10^{9}$ pairs. It is therefore tempting to deduce that an order of 54,300 neurons (which are $1.47 \times 10^{9}$ pairs) share inputs that induce pauses. This number is strikingly high given the rate of divergence for the main inhibitory inputs of the GPe, the striatal MSNs. Each of these neurons creates $\sim 200-$ 300 synapses in the GPe, usually confined to very few $(<10)$ neurons that receive multiple inputs from the same MSN (Yelnik et al., 1996). This suggests that the network pauses we observed could be produced by synchronized activation from many striatal MSNs. The other major input to the GPe comes from the STN. The influence of these neurons on the GPe is far less specific than that of the MSNs (Parent and Hazrati, 1995), suggesting that it might not be an increase in MSN activity, but rather a reduction in the STN activity, that is the main driving force of pauses (Nambu et al., 2000). Either way, we believe more light can be shed on the issue given simultaneous recordings of GPe cells and MSNs or STN cells. Notably, these estimates could be influenced by the fact that our simultaneously recorded neurons were located in relatively close proximity to one another $(<1.5 \mathrm{~mm})$, influencing coincidence significance in a manner that does not represent the whole GPe.

Our finding that numerous pairs show coincidence levels higher than chance is somewhat negated by the relatively low coincidence indices, indicating that even pairs that pause together unquestionably do so in a very nondeterministic manner (Fig. $4 a$, left). The reason for this stochasticity remains unclear. Two likely explanations include either a dependency of the pause response on the intrinsic state of a neuron or a dependency of pauses on several congruent inputs arriving in a temporally precise way. Both of these avenues require further modeling and experimentation. However, it is worthwhile acknowledging that even small coincidence metrics might go a long way, as has been shown for pairwise correlations between neurons in different brain areas (Schneidman et al., 2003, 2006).

In conclusion, our findings shed light on the origin and features of pausing behavior in the GPe, but leave the riddle concerning their function and downstream effects mostly untouched. These silences in spiking behavior, first described over 40 years ago, are proba- bly the key characteristic feature of the GPe, the central nucleus of the basal ganglia network, yet they have received relatively little focus in recent research. We believe that such focus could result in a better understanding of the basal ganglia as a whole, and its role in normal behavior and in disease.

\section{References}

Adler A, Joshua M, Rivlin-Etzion M, Mitelman R, Marmor O, Prut Y, Bergman H (2010) Neurons in both pallidal segments change their firing properties similarly prior to closure of the eyes. J Neurophysiol 103:346359. CrossRef Medline

Adler A, Katabi S, Finkes I, Israel Z, Prut Y, Bergman H (2012) Temporal convergence of dynamic cell assemblies in the striato-pallidal network. J Neurosci 32:2473-2484. CrossRef Medline

Adler A, Finkes I, Katabi S, Prut Y, Bergman H (2013a) Encoding by synchronization in the primate striatum. J Neurosci 33:4854-4866. CrossRef Medline

Adler A, Katabi S, Finkes I, Prut Y, Bergman H (2013b) Different correlation patterns of cholinergic and GABAergic interneurons with striatal projection neurons. Front Syst Neurosci 7:47. CrossRef Medline

Chiken S, Shashidharan P, Nambu A (2008) Cortically evoked long-lasting inhibition of pallidal neurons in a transgenic mouse model of dystonia. J Neurosci 28:13967-13977. CrossRef Medline

Day M, Wokosin D, Plotkin JL, Tian X, Surmeier DJ (2008) Differential excitability and modulation of striatal medium spiny neuron dendrites. J Neurosci 28:11603-11614. CrossRef Medline

DeLong MR (1971) Activity of pallidal neurons during movement. J Neurophysiol 34:414-427. Medline

DeLong MR, Crutcher MD, Georgopoulos AP (1985) Primate globus pallidus and subthalamic nucleus: functional organization. J Neurophysiol 53:530-543. Medline

Difiglia M, Pasik P, Pasik T (1982) A Golgi and ultrastructural study of the monkey globus pallidus. J Comp Neurol 212:53-75. CrossRef Medline

Elias S, Joshua M, Goldberg JA, Heimer G, Arkadir D, Morris G, Bergman H (2007) Statistical properties of pauses of the high-frequency discharge neurons in the external segment of the globus pallidus. J Neurosci 27: 2525-2538. CrossRef Medline

François C, Percheron G, Yelnik J, Heyner S (1984) A Golgi analysis of the primate globus pallidus. I. Inconstant processes of large neurons, other neuronal types, and afferent axons. J Comp Neurol 227:182-199. CrossRef Medline

Gerfen CR, Engber TM, Mahan LC, Susel Z, Chase TN, Monsma FJ Jr, Sibley DR (1990) D1 and D2 dopamine receptor-regulated gene expression of striatonigral and striatopallidal neurons. Science 250:1429-1432. CrossRef Medline

Gittis AH, Berke JD, Bevan MD, Chan CS, Mallet N, Morrow MM, Schmidt R (2014) New roles for the external globus pallidus in basal ganglia circuits and behavior. J Neurosci 34:15178-15183. CrossRef Medline

Goldberg JA, Bergman H (2011) Computational physiology of the neural networks of the primate globus pallidus: function and dysfunction. Neuroscience 198:171-192. CrossRef Medline

Hashimoto K, Kita H (2006) Slow oscillatory activity of rat globus pallidus neurons in vitro. Eur J Neurosci 23:443-453. CrossRef Medline

Hodgkin AL (1948) The local electric changes associated with repetitive action in a non-medullated axon. J Physiol 107:165-181. CrossRef Medline

Izhikevich EM (2007) Dynamical systems in neuroscience: the geometry of excitability and bursting. Cambridge, MA: MIT.

Jaeger D, Kita H (2011) Functional connectivity and integrative properties of globus pallidus neurons. Neuroscience 198:44-53. CrossRef Medline

Joshua M, Elias S, Levine O, Bergman H (2007) Quantifying the isolation quality of extracellularly recorded action potentials. J Neurosci Methods 163:267-282. CrossRef Medline

Kaneda K, Kita H (2005) Synaptically released GABA activates both preand postsynaptic $\mathrm{GABA}(\mathrm{B})$ receptors in the rat globus pallidus. J Neurophysiol 94:1104-1114. CrossRef Medline

Kita H, Nambu A, Kaneda K, Tachibana Y, Takada M (2004) Role of ionotropic glutamatergic and GABAergic inputs on the firing activity of neurons in the external pallidum in awake monkeys. J Neurophysiol 92:3069-3084. CrossRef Medline

Kita H, Tachibana Y, Nambu A, Chiken S (2005) Balance of monosynaptic excitatory and disynaptic inhibitory responses of the globus pallidus in- 
duced after stimulation of the subthalamic nucleus in the monkey. J Neurosci 25:8611-8619. CrossRef Medline

Kita H, Chiken S, Tachibana Y, Nambu A (2006) Origins of $\mathrm{GABA}_{\mathrm{A}}$ and $\mathrm{GABA}_{\mathrm{B}}$ receptor-mediated responses of globus pallidus induced after stimulation of the putamen in the monkey. J Neurosci 26:6554-6562. CrossRef Medline

Legéndy CR, Salcman M (1985) Bursts and recurrences of bursts in the spike trains of spontaneously active striate cortex neurons. J Neurophysiol 53: 926-939. Medline

Mallet N, Micklem BR, Henny P, Brown MT, Williams C, Bolam JP, Nakamura KC, Magill PJ (2012) Dichotomous organization of the external globus pallidus. Neuron 74:1075-1086. CrossRef Medline

Nambu A, Tokuno H, Hamada I, Kita H, Imanishi M, Akazawa T, Ikeuchi Y, Hasegawa N (2000) Excitatory cortical inputs to pallidal neurons via the subthalamic nucleus in the monkey. J Neurophysiol 84:289-300. Medline

Parent A, Hazrati LN (1995) Functional anatomy of the basal ganglia. II. The place of subthalamic nucleus and external pallidum in basal ganglia circuitry. Brain Res Brain Res Rev 20:128-154. CrossRef Medline

Percheron G, François C, Yelnik J, Fénelon G, Talbi B (1994) The basal ganglia related system of primates: definition, description and informational analysis. In: The basal ganglia IV: new ideas and data on structure and function (Percheron G, McKenzie J, Féger J, eds), pp 3-20. New York: Springer US.

Sadek AR, Magill PJ, Bolam JP (2007) A single-cell analysis of intrinsic con- nectivity in the rat globus pallidus. J Neurosci 27:6352-6362. CrossRef Medline

Sato F, Lavallée P, Lévesque M, Parent A (2000) Single-axon tracing study of neurons of the external segment of the globus pallidus in primate. J Comp Neurol 417:17-31. CrossRef Medline

Schneidman E, Still S, Berry MJ 2nd, Bialek W (2003) Network information and connected correlations. Phys Rev Lett 91:238701. CrossRef Medline

Schneidman E, Berry MJ 2nd, Segev R, Bialek W (2006) Weak pairwise correlations imply strongly correlated network states in a neural population. Nature 440:1007-1012. CrossRef Medline

Shink E, Smith Y (1995) Differential synaptic innervation of neurons in the internal and external segments of the globus pallidus by the GABA- and glutamate-containing terminals in the squirrel monkey. J Comp Neurol 358:119-141. CrossRef Medline

Surmeier DJ, Carrillo-Reid L, Bargas J (2011) Dopaminergic modulation of striatal neurons, circuits, and assemblies. Neuroscience 198:3-18. CrossRef Medline

Yelnik J, Percheron G, François C (1984) A Golgi analysis of the primate globus pallidus. II. Quantitative morphology and spatial orientation of dendritic arborizations. J Comp Neurol 227:200-213. CrossRef Medline

Yelnik J, François C, Percheron G, Tandé D (1996) A spatial and quantitative study of the striatopallidal connection in the monkey. Neuroreport 7:985-988. CrossRef Medline 